\title{
Association between endoscopic findings of eosinophilic esophagitis and responsiveness to proton pump inhibitors
}

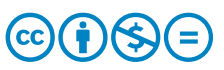

\author{
Authors \\ Tetsuya Tanigawa, Toshio Watanabe, Yasuhiro Fujiwara \\ Institution \\ Department of Gastroenterology, Osaka City University \\ Graduate School of Medicine \\ submitted 15.10.2018 \\ accepted after revision 29.1.2019 \\ Bibliography \\ DOI https://doi.org/10.1055/a-0859-7276 | \\ Endoscopy International Open 2019; 07: E433-E439 \\ (c) Georg Thieme Verlag KG Stuttgart · New York \\ ISSN 2364-3722 \\ Corresponding author \\ Akinari Sawada, MD, PhD, 1-4-3 Asahimachi, Abenoku, \\ Osaka 545-8585, Japan \\ Fax: $+81-666453813$ \\ m1164972@med.osaka-cu.ac.jp
}

Akinari Sawada, Atsushi Hashimoto, Risa Uemura, Koji Otani, Fumio Tanaka, Yasuaki Nagami, Hirokazu Yamagami,

\section{ABSTRACT}

Background and study aims Endoscopic findings of esophageal eosinophilia sometimes localize to small areas of the esophagus. A previous study suggested that pathogenesis of localized-type eosinophilic esophagitis (LEoE) was associated with acid reflux. However, LEoE treatment outcomes have not been studied. We aimed to analyze the clinical and histologic significance of LEoE in comparison with diffuse-type eosinophilic esophagitis (DEoE).

Patients and methods This study included 106 patients with esophageal eosinophilia. Esophageal eosinophilia was defined as a condition where the maximum number of intraepithelial eosinophils was $\geq 15$ per high-power field. LEoE was defined as an endoscopic lesion confined to onethird of the esophagus: upper, middle, or lower. Esophageal eosinophilia encompassing more than two-thirds of the esophagus was defined as DEoE. We retrospectively compared LEoE and DEoE in terms of clinical characteristics, histologic findings, and proportion of proton pump inhibitor (PPI) responders.

Results Of 106 patients, 12 were classified as having LEoE and 94 were classified as having DEoE. The proportion of asymptomatic patients was significantly higher in the LEoE group than the DEoE group ( $42 \%$ vs $7 \%, P<0.01$ ). In the LEoE group, 10 patients ( $84 \%$ ) had endoscopic lesions in the lower esophagus. The maximum number of eosinophils did not differ between the groups (54 [24-71] for LEoE, 40 [20 - 75] for DEoE, $P=0.65$ ). The prevalence of PPI responders was significantly higher in the LEoE group than the DEoE group ( $100 \%$ vs $63 \%, P=0.01$ ).

Conclusion LEoE can be a sign of good responsiveness to PPI therapy.

\section{Introduction}

Eosinophilic esophagitis (EoE) is a chronic, local immune-mediated esophageal disease clinically characterized by symptoms related to esophageal dysfunction and histologically characterized by eosinophil-predominant inflammation [1]. EoE is diagnosed based on symptoms, such as dysphagia, food impaction, heartburn, chest pain, and intraepithelial eosinophil infiltration of $\geq 15$ per high power field (hpf). Several studies showed a steady increase in incidence and prevalence rates of EoE in western countries [2,3].

Approximately $50 \%$ to $60 \%$ of EoE patients are effectively treated with proton pump inhibitors (PPI), but increased esoph- ageal acid exposure on $\mathrm{pH}$ monitoring was not associated with the efficacy of PPI therapy [4]. In fact, many reports revealed that PPI responders and PPI non-responders had common characteristics in many aspects [5].

EoE often has characteristic endoscopic findings, such as linear furrows, rings, white exudates, and strictures [6]. Although these findings are usually found distributed throughout the entire esophagus, Abe et al. reported some cases of EoE with endoscopic findings localized to a small part of the esophagus, especially above the esophagogastric junction (EG]) [7]. The authors suggested that gastric acid reflux might be involved with this type of esophageal eosinophilia. However, it remains unknown to what extent PPI ameliorates localized-type EoE (LEoE). 


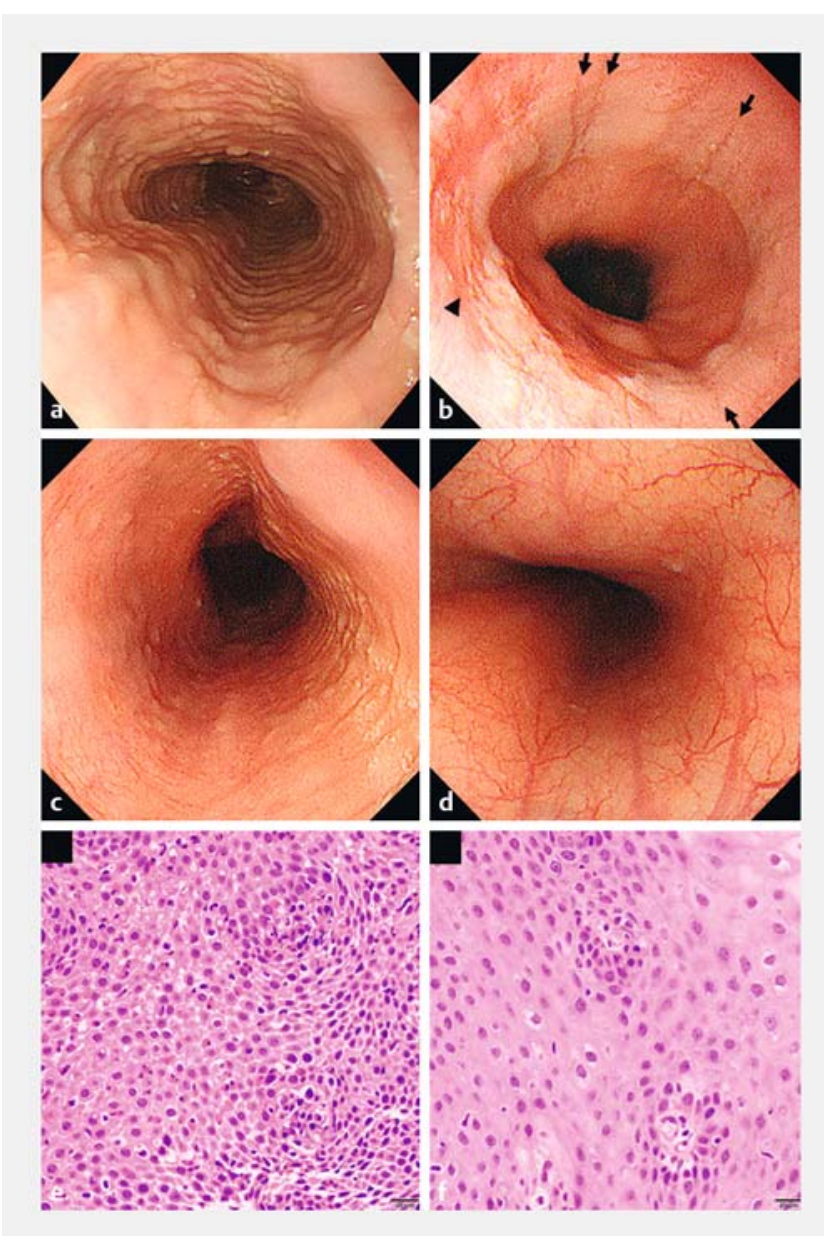

- Fig. 1 Endoscopic and histologic findings of eosinophilic esophagitis. a Endoscopic findings of DEoE. Rings, white exudates, and linear furrows were observed in the etire esophagus. b Case 1 of LEoE. White turbidity was observed along the entire circumference just above the EGJ. In addition, linear furrows (black arrows) and white exudates (arrowhead) were distributed on white turbidity. c Case 2 of LEoE. White exudates and subtle rings were located along only half of the circumference of the upper esophagus. d Normal esophageal mucosa was found from the EGJ to middle esophagus. e, $\mathbf{f}$ Histologic findings in Case 2. e Esophageal biopsy samples taken from the mucosa with endoscopic findings around image $\mathbf{c}$ showed $>15$ eosinophils/hpf, other inflammatory cells, and dilated intracellular space in the esophageal epithelium. f In contrast, a sample taken from the mucosa without endoscopic findings around image $\mathbf{d}$ showed no intraepithelial eosinophils. LEoE, localized-type eosinophilic esophagitis; DEoE, diffuse-type eosinophilic esophagitis; EG], esophagogastric junction.

In this study, we aimed to retrospectively investigate clinical and histological characteristics of LEoE including treatment outcomes.

\section{Patients and methods}

\section{Patients and study designs}

A total of 115 patients who had esophageal eosinophilia found by esophagogastroduodenoscopy (EGD) for medical check-up or investigation of gastrointestinal symptoms were referred to
Osaka City University Hospital between April 2010 and February 2018. Esophageal eosinophilia was defined as an intraepithelial eosinophilic infiltration $\geq 15$ per hpf in at least one biopsy sample. Of 115 patients, six patients with eosinophilic gastroenteritis and three patients lost during the follow-up period were excluded. The remaining 106 patients were initially treated with PPIs or steroids. An EGD was then performed to take at least five esophageal biopsies to assess the intraepithelial eosinophilic infiltration using Single-Use Radial Jaw 4TM (Boston, Scientific Japan, Tokyo, Japan). The biopsies were taken as follows: two from the distal esophagus, two from the middle esophagus, and one or two from the proximal esophagus. Biopsy samples were fixed with $10 \%$ buffered formalin, embedded in paraffin blocks, and stained with hematoxylin and eosin. The number of eosinophils was counted using an optical microscope. Patients' clinical characteristics were retrospectively analyzed and included age, sex, body mass index (BMI), smoking and alcohol habits, comorbidities of allergic diseases, and blood tests measuring peripheral eosinophil counts and Helicobacter pylori antigen. Endoscopic findings at diagnosis and after the first treatment were also evaluated. This study was approved by the Ethical Committee of Osaka City University Medical School.

\section{Classification of esophageal eosinophilia: localized or diffuse-type EoE}

Patients were divided into two groups based on endoscopic findings of esophageal eosinophilia regardless of histological eosinophilic distribution in the esophagus as described in the previous report: (1) rings defined as a corrugated appearance or as trachealization; (2) white plaques which appeared as punctate white nodules, dispersant flocculant material, or in a granular pattern; (3) linear furrows representative of mucosal edema and thickening with vertical lines along the length of the esophageal mucosa; and (4) strictures [7]. LEoE was defined as having any of these findings in any one-third of the esophagus: upper, middle, and lower ( $\triangleright$ Fig.1). Diffuse-type EoE (DEoE) was defined as endoscopic lesions extending through more than one-third of the esophagus ( $\mathbf{F i g . 1}$ ). In the LEoE group, distribution of endoscopic lesions was evaluated, and all endoscopic images were reviewed by a single experienced endoscopist (A.S.).

\section{Reflux esophagitis, atrophic gastritis, and hiatal hernia}

Reflux esophagitis was evaluated using the Los Angeles classification [8]. If a patient had a mucosal break of grade A or more, the patient was considered to have reflux esophagitis. Atrophic gastritis was classified by the Kimura-Takemoto classification [9]. Patients were considered to have atrophic gastritis when they had grade C-2 (the atrophic border was on the lesser curvature of the body) or more. Hiatal hernia was classified by the Hill's classification [10]. Patients were considered to have hiatal hernia when they had grade 2 or more. 
- Table 1 Clinical characteristics in each endoscopic type of eosinophilic esophagitis.

\begin{tabular}{|c|c|c|c|}
\hline & $\begin{array}{l}\text { LeoE } \\
(N=12)\end{array}$ & $\begin{array}{l}\text { DeoE } \\
(N=94)\end{array}$ & $P$ value \\
\hline Age at diagnosis (median, IQR) & $44(40-51)$ & $46(40-52)$ & 0.66 \\
\hline Male (n, \%) & $9(75)$ & $60(64)$ & 0.54 \\
\hline BMI $\left(\mathrm{kg} / \mathrm{m}^{2}\right)$ (median, IQR) & $23.1(21.1-24.7)$ & $23.3(21.3-26.4)$ & 0.48 \\
\hline Allergy history (n, \%) & $8(67)$ & $67(71)$ & 0.75 \\
\hline Smoking (n, \%) & $1(8)$ & $10(11)$ & 1 \\
\hline Alcohol (n, \%) & $6(50)$ & $26(28)$ & 0.18 \\
\hline H. pylori infection (n, \%) & $1(10)$ & $21(31)$ & 0.27 \\
\hline Steroid use at diagnosis & $0(0)$ & $3(3)$ & 1 \\
\hline \multicolumn{4}{|l|}{ Symptoms (n, \%) } \\
\hline - Dysphagia & $4(33)$ & $69(73)$ & 0.01 \\
\hline - Heartburn & $3(25)$ & $23(24)$ & 1 \\
\hline - Chest pain & $3(25)$ & $13(14)$ & 0.39 \\
\hline - Any of three symptoms & $7(58)$ & $87(93)$ & 0.004 \\
\hline \multicolumn{4}{|l|}{ Blood test (median, IQR) } \\
\hline - WBC (/pl) & $5600(5375-5850)$ & $5500(4750-6600)$ & 0.80 \\
\hline - Number of Eosinophils (/ $\mu \mathrm{L})$ & $269(230-393)$ & $270(180-420)$ & 0.85 \\
\hline - Proportion of Eosinophils (\%) & $4.8(4.0-7.0)$ & $5.0(3.7-7.8)$ & 0.98 \\
\hline - $\lg E(I U / m L)$ & $94(77.0-200)$ & $180(54.5-383)$ & 0.27 \\
\hline \multicolumn{4}{|l|}{ Primary treatment } \\
\hline . PPI (n, \%) & $11(92)$ & $90(96)$ & 0.46 \\
\hline - Topical steroid (n, \%) & $0(0)$ & $3(3)$ & 1 \\
\hline - Systemic steroid (n, \%) & $0(0)$ & $1(1)$ & 1 \\
\hline - $\operatorname{ESD}(n, \%)$ & $1(8)$ & $0(0)$ & 0.11 \\
\hline \multicolumn{4}{|c|}{$\begin{array}{l}\text { Data were expressed as the median (interquartile range) or numbers with frequencies. LEoE, localized-type eosinophilic esophagitis; DEoE, diffuse-type eosinophil } \\
\text { esophagitis; IQR, interquartile range; PPI, proton pump inhibitor; BMI, body mass index; H. pylori, Helicobacter pylori; WBC, white blood cell; ESD, endoscopic sub- } \\
\text { mucosal dissection. }\end{array}$} \\
\hline
\end{tabular}

\section{Assessment of responsiveness to PPI therapy}

Patients were defined as a PPI responder when their intraepithelial eosinophilic infiltration decreased to less than 15 per hpf and symptoms were completely relieved after 8 weeks of PPI therapy.

\section{Statistical analyses}

Data were expressed as the median (interquartile range) in continuous variables and as numbers and frequencies in categorical variables. The Mann-Whitney $U$ test was used for continuous variables. Fisher's exact test was used for categorical variables. Statistical analyses were performed using the R statistical package. $P<0.05$ was considered statistically significant.

\section{Results}

\section{Study patients}

We analyzed a total of 106 patients with esophageal eosinophilia and a median age of 46 years (range $41-52$ ). The majority of patients were male (65\%), and most patients (89\%) had symptoms including dysphagia (69\%), heartburn (25\%), and chest pain (15\%). Seventy-four patients (70\%) had comorbidities of allergic diseases as follows: 20 patients (19\%) with asthma, 42 patients (40\%) with rhinitis, 12 patients $(11 \%)$ with atopic dermatitis, and 24 patients (23\%) with food allergies.

Based on the distribution of endoscopic lesions, 12 patients were assigned to the LEoE group, and the remaining 94 patients were assigned to the DEoE group.Clinical characteristics of each group are shown in $>$ Table 1 . There were no significant differences with regard to age, sex, BMI, prevalence of allergic diseases, smoking and drinking habit, and infection rate of 
- Table 2 Endoscopic and histologic findings of each endoscopic type of eosinophilic esophagitis.

\begin{tabular}{|c|c|c|c|}
\hline & $\begin{array}{l}\text { LeoE } \\
(N=12)\end{array}$ & $\begin{array}{l}\text { DeoE } \\
(N=94)\end{array}$ & $P$ value \\
\hline \multicolumn{4}{|l|}{ Endoscopic findings } \\
\hline \multicolumn{4}{|l|}{ - Prevalence rate of each EoE finding } \\
\hline - Rings (n, \%) & $1(8)$ & $38(40)$ & 0.05 \\
\hline - White plaques (n, \%) & $8(67)$ & $62(66)$ & 1 \\
\hline - Linear furrows (n, \%) & $9(75)$ & $90(96)$ & 0.03 \\
\hline - Stricture (n, \%) & $0(0)$ & $1(1)$ & 1 \\
\hline \multicolumn{4}{|l|}{ - Location of endoscopic findings of EoE } \\
\hline - Upper esophagus (n, \%) & $2(16)$ & & \\
\hline - Mid esophagus (n, \%) & $0(0)$ & & \\
\hline - Lower esophagus (n, \%) & $5(42)$ & & \\
\hline - Just Around EGJ (n, \%) & $5(42)$ & & \\
\hline - Reflux esophagitis (n, \%) & $1(8)$ & $23(25)$ & 0.29 \\
\hline - Grade A & $1(8)$ & $19(21)$ & \\
\hline - Grade B & $0(0)$ & $3(3)$ & \\
\hline - Grade C & $0(0)$ & $1(1)$ & \\
\hline - Atrophic gastritis (n, \%) & $2(17)$ & $18(20)$ & 1 \\
\hline - Hiatal hernia (n, \%) & $1(8)$ & $10(11)$ & 1 \\
\hline \multicolumn{4}{|l|}{ Histologic findings (median, IQR) } \\
\hline - Number of biopsies & $3(2-5)$ & $5(2-6)$ & 0.10 \\
\hline - Maximum number of eosinophils (/hpf) & $54(24-71)$ & $40(20-75)$ & 0.65 \\
\hline
\end{tabular}

H. pylori between the LEoE and DEoE groups. The proportion of symptomatic patients in the LEoE group was significantly lower compared to the DEoE group ( $58 \%$ for LEoE, $93 \%$ for DEoE, $P=$ 0.004). Blood tests did not show any difference between the two groups in the number and proportion of eosinophils, and serum concentration of $\mathrm{IgE}$. There were also no differences in these parameters between the two groups with symptoms.

Of 106 patients, 101 patients (95\%) were treated with a standard dose of PPI for 8 weeks, regardless of whether they had symptoms related to esophageal dysfunction, and four severe symptomatic patients $(4 \%)$ were treated with steroids. One patient with LEoE (1\%) was diagnosed after endoscopic submucosal dissection (ESD) of the lesion ( $\downarrow$ Table 1 ).

\section{Endoscopic and histologic findings}

Compared to the DEoE group, the LEoE group had significantly fewer linear furrows (75\% for $\mathrm{LEOE}, 96 \%$ for DEoE, $P=0.03$ ), but the prevalence of other endoscopic findings such as rings, white plaques, and strictures did not differ between the two groups ( $\triangleright$ Table 2 ). Ten patients (84\%) in the LEoE group had endoscopic lesions in the lower esophagus. Among them, the endoscopic lesions of five patients (42\%) were located around the EGJ, within $1 \mathrm{~cm}$, proximal from the squamocolumnar junction; two patients (16\%) had lesions in the upper esophagus ( $\triangleright$ Table 2). In the LEoE group, endoscopic lesions were distributed throughout the entire circumference of the esophagus in seven patients (58\%) and covered $50 \%$ or less of the circumference in five patients (42\%). Prevalence of reflux esophagitis, atrophic gastritis, and hiatal hernia did not significantly differ between the 2 groups ( $>$ Table 2 ).

As for the histologic findings, there were no significant differences in either the number of biopsies taken for diagnosis or the maximum number of eosinophils per hpf in the esophageal epithelium between the two groups ( $>$ Table 2 ). Endoscopic and histologic findings did not differ between the two groups when analyzing symptomatic patients.

In the LEoE group, five patients (42\%) had a biopsy taken from the mucosa both with and without endoscopic lesions: 15 samples were taken from the mucosa without endoscopic lesions and nine samples were taken from the mucosa with endoscopic lesions. The maximum number of eosinophils was significantly higher in the samples with endoscopic lesions than in those without endoscopic lesions (70 [20 - 79] for sam- 


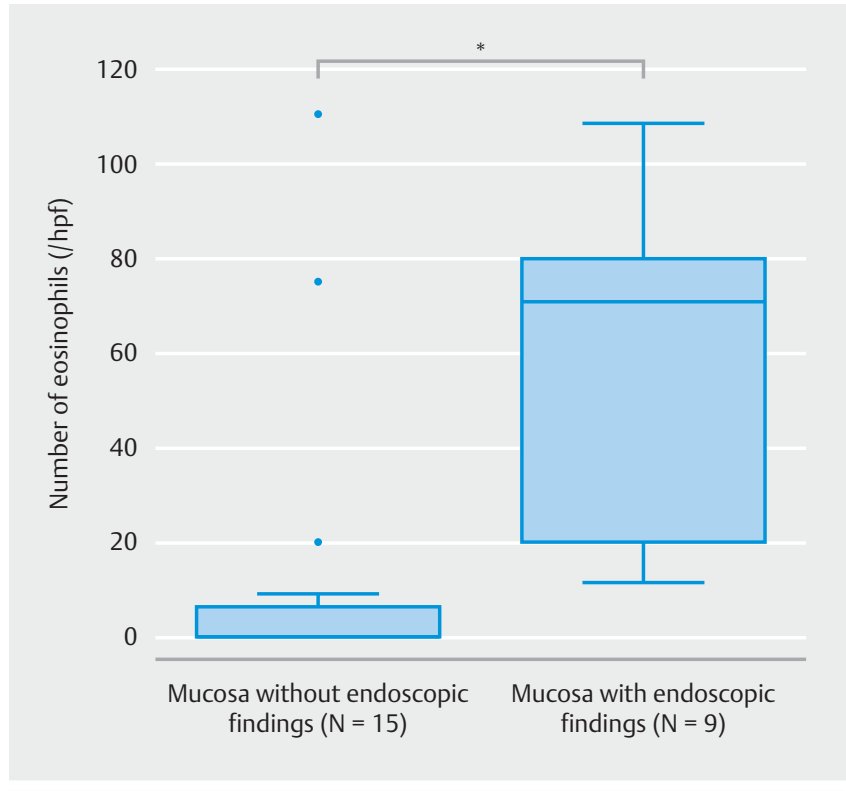

- Fig. 2 Number of eosinophils in each esophageal biopsy sample with or without endoscopic findings in localized-type eosinophilic esophagitis. ${ }^{*} P<0.01$.

ples with endoscopic lesions, 0 [0-6.5] for those without endoscopic lesions, $P<0.01$ ) ( $>$ Fig. 2 ).

\section{Response to PPI therapy}

In patients who received 8-week PPI therapy, all 11 in the LEoE group who had alleviation of lesions histologically and were symptomatic became asymptomatic, whereas 57 of 90 patients $(63 \%)$ in the DEoE group responded to PPI therapy, showing a significant difference between the two groups $(P=0.01)$ ( $>$ Fig.3). Even though we analyzed only symptomatic patients, the rate of response to PPI between the two groups still differed significantly (7 out of 7 [100\%] for the LEoE group, 51 out of 83 [61\%] for the DEoE group, $P=0.047)$. Of 11 asymptomatic patients in both groups, 10 patients $(91 \%)$ had histologic amelioration with PPI therapy.

\section{Discussion}

EoE has patchy distribution of eosinophil infiltration [11], however, characteristic endoscopic findings of EoE are usually found diffusely in the esophagus. Although EoE with localized endoscopic findings is occasionally detected, its significance has not been elucidated. There has been only one study, by Abe et al., that has reported characteristics of LEoE [7], in which they assumed that acid reflux caused esophageal inflammation, increasing the permeability of the esophageal mucosa to causal antigens. If that hypothesis is the case, PPI would be more likely to improve LEoE than DEoE, in which the influence of acid reflux on pathophysiology varies. The efficacy of PPI for LEoE has not been studied, therefore, we conducted this study.

A couple of guidelines for EoE specifically reference PPI responders, or so-called PPI-responsive esophageal eosinophilia (PPI-REE), as a different entity than EoE or as gastroesophageal

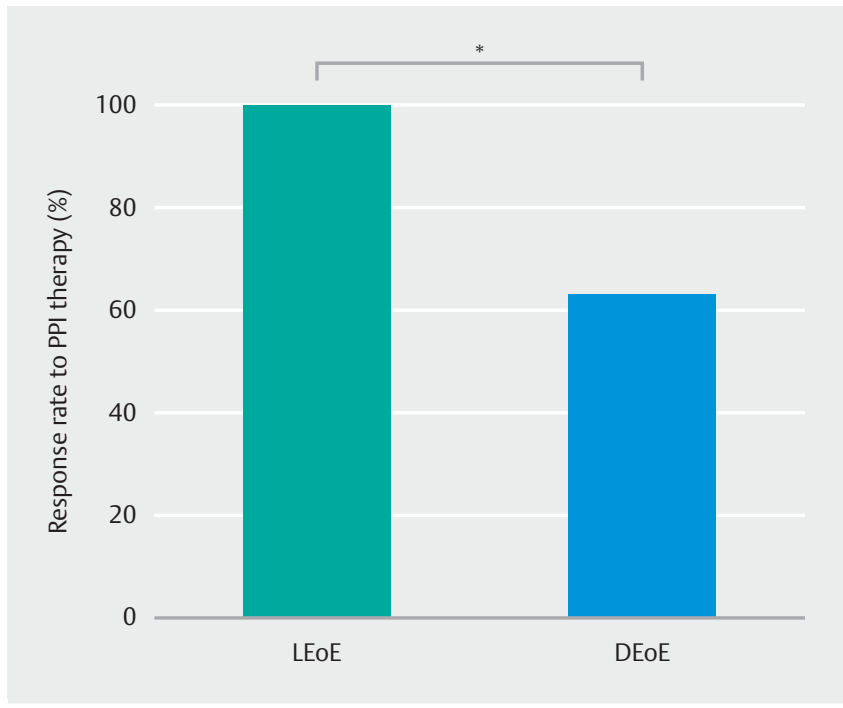

- Fig. 3 Response rate to PPI therapy of each type of eosinophilic esophagitis. LEoE, localized-type eosinophilic esophagitis; DEoE, diffuse-type eosinophilic esophagitis. ${ }^{*} P<0.05$.

reflux disease (GERD)-induced esophageal eosinophilia [12, 13]. After these guidelines had been published, many reports showed that EoE and PPI-REE could not be distinguished clearly in several aspects including endoscopic findings [14-18]. Moreover, it is unrealistic to exclude GERD from EoE because GERD may coexist and interact bi-directionally [19], even though GERD is definitely a different disease than EoE [5]. Consequently, the latest guidelines from United European Gastroenterology and the American Gastroenterological Association treat PPI-REE as part of the EoE continuum $[1,20]$. Every guideline proposes that EoE be defined by symptoms related to esophageal dysfunction $[1,12,13,21]$; nevertheless, we included asymptomatic patients to elucidate their clinical significance, and they accounted for $11 \%$ of studied patients,. However, most results including PPI response did not change even when analyzing only symptomatic patients.

This study showed that the LEoE group had clinical characteristics similar to the DEoE group, but more of the the LEoE group were asymptomatic. These findings correspond with the previous report [7], although patients with reflux esophagitis were not included in their study. There is no consistent evidence showing an association of endoscopic findings with clinical and histological activity. Some reports showed that the grade of endoscopic findings using the Endoscopic Reference Score (EREFS) [6] did not adequately predict the number of intraepithelial eosinophils and symptom severity [22,23], but Dellon et al. showed a good correlation between EREFS and histologic findings in EoE [24]. EREFS does not adopt the area of comprehensive endoscopic findings as one criterion, so it is difficult to compare LEoE to DEoE using EREFS. However, the less comprehensive endoscopic findings in LEoE are likely to be associated with a higher proportion of asymptomatic patients. This study proved that eosinophil infiltration in the LEoE group was to the same degree as that in the DEoE group, but was localized in accordance with endoscopic findings. This result con- 
formed to a larger meta-analysis study that reported approximately $80 \%$ to $90 \%$ of subjects with endoscopically normal findings have no pathological number of intraepithelial eosinophils [25]. Therefore, the degree of inflammation in the whole esophagus could be milder in LEoE than in DEoE, which might be relevant to symptom generation.

A couple of population-based studies showed a prevalence of asymptomatic subjects with esophageal eosinophilia, but the natural history of this condition has not been elucidated $[26,27]$. Because there is a possibility that asymptomatic esophageal eosinophilia could be the halfway point in the development of EoE, an early intervention could contribute to prevention of stricture formation [28]. Therefore, we attempted to treat asymptomatic patients with esophageal eosinophilia, even though a treatment consensus for these patients has not been established. This study showed that all patients in the LEoE group responded to PPI therapy, and the response rate was statistically superior to that of the DEoE group. This result did not change, even when only symptomatic patients were included in the analysis, and the proportion of PPI responders among the symptomatic patients (67\%) was acceptable in comparison to other studies [4, 29].

Given that PPIs have two effects on the gastrointestinal tract, which are gastric acid suppression and anti-inflammatory effects by multiple mechanisms [30,31], two possible scenarios could be considered to explain why PPIs significantly improved LEoE. First, gastric acid might contribute more to pathogenesis in the LEoE group compared to the DEoE group. In fact, the LEoE group had endoscopic findings primarily in the lower esophagus, including areas near the EG], which was consistent with a previous report [7]. Because acid reflux can induce an increase in esophageal permeability leading to antigen penetration of the epithelium [19], this hypothesis seems reasonable. However, further study using $\mathrm{pH}$ meters should be performed. Second, the LEoE group might be a lower-disease activity phenotype of EoE. We found two LEoE cases with upper esophageal lesions never seen in the previous study [7]. It is unlikely that these two cases were involved with acid reflux; therefore, it is possible that the anti-inflammatory effect rather than the anti-acid effect of PPIs influenced them [30,32,33]. LEoE had small areas damaged by eosinophil-induced inflammation in this study, both endoscopically and histologically, which may explain why the LEoE group responded better to the anti-inflammatory effect of PPI.

Many cancers including esophageal squamous carcinoma (ESC) accompany eosinophil infiltration, which is associated with an improved prognosis [34,35]. However, to the best of our knowledge, this is the first case of EoE mimicking ESC. The endoscopic lesion resembled EoE, but a couple of factors, including the localized nature of the lesion and the pathological diagnosis for a biopsy contributed to consider it as ESC. After the resected area healed, several biopsies taken from both the scar and other esophageal mucosa showed an absence of eosinophils without PPI use. Localized endoscopic lesions with features of EoE warrant careful attention when the biopsy sample shows possible evidence of a malignancy.
This study has some limitations. First, the sample size was small, and the study design was retrospective. However, as LEoE was a relatively rare type of esophageal eosinophilia, it was difficult to perform a prospective study. Second, there might be referral bias, because this study was performed in a tertiary medical center. This study included a higher proportion of symptomatic patients than the previous report [7]. Thus, further investigation with more cases is required.

\section{Conclusion}

In conclusion, LEoE accounted for approximately $10 \%$ of esophageal eosinophilia. All LEoE were clinically and histologically improved by PPI therapy, and most LEoE had endoscopic findings in the lower esophagus. These findings suggest that gastric acid is more relevant to pathogenesis of $L E o E$ than $D E o E$, and LEoE is a less-active phenotype of EoE. Endoscopic findings that show LEoE prior to any treatment are likely to predict a good response to PPI therapy, and can sometimes mimic ESC because of its localization.

\section{Competing interests}

None

References

[1] Lucendo AJ, Molina-Infante J, Arias Á et al. Guidelines on eosinophilic esophagitis: evidence-based statements and recommendations for diagnosis and management in children and adults. United European Gastroenterol J 2017; 5: 335-358

[2] Dellon ES, Hirano I. Epidemiology and natural history of eosinophilic esophagitis. Gastroenterology 2018; 154: 319-332.e313

[3] Arias Á, Pérez-Martínez I, Tenías JM et al. Systematic review with meta-analysis: the incidence and prevalence of eosinophilic oesophagitis in children and adults in population-based studies. Aliment Pharmacol Ther 2016; 43: 3-15

[4] Lucendo AJ, Arias Á, Molina-Infante J. Efficacy of proton pump inhibitor drugs for inducing clinical and histologic remission in patients with symptomatic esophageal eosinophilia: a systematic review and meta-analysis. Clin Gastroenterol Hepatol 2016; 14: 13-22.e11

[5] Molina-Infante J, Bredenoord AJ, Cheng E et al. Proton pump inhibitorresponsive oesophageal eosinophilia: an entity challenging current diagnostic criteria for eosinophilic oesophagitis. Gut 2016; 65: 524 531

[6] Hirano I, Moy N, Heckman MG et al. Endoscopic assessment of the oesophageal features of eosinophilic oesophagitis: validation of a novel classification and grading system. Gut 2013; 62: 489-495

[7] Abe Y, lijima K, Ohara S et al. Localized esophageal eosinophilia: Is it an early manifestation of eosinophilic esophagitis or a subtype of gastroesophageal reflux disease? Dig Endosc 2014; 26: 337-343

[8] Lundell LR, Dent J, Bennett JR et al. Endoscopic assessment of oesophagitis: clinical and functional correlates and further validation of the Los Angeles classification. Gut 1999; 45: 172-180

[9] Kimura K, Takemoto T. An endoscopic recognition of the atrophic border and its significance in chronic gastritis. Endoscopy 1969; 1: $87-97$ 
[10] Hill LD, Kozarek RA, Kraemer S] et al. The gastroesophageal flap valve: in vitro and in vivo observations. Gastrointest Endosc 1996; 44: 541 547

[11] Saffari H, Peterson KA, Fang JC et al. Patchy eosinophil distributions in an esophagectomy specimen from a patient with eosinophilic esophagitis: Implications for endoscopic biopsy. J Allergy Clin Immunol 2012; 130: $798-800$

[12] Liacouras CA, Furuta GT, Hirano I et al. Eosinophilic esophagitis: updated consensus recommendations for children and adults. J Allergy Clin Immunol 2011; 128: 3-20.e26; quiz 21-22

[13] Dellon ES, Gonsalves N, Hirano I et al. ACG clinical guideline: Evidenced based approach to the diagnosis and management of esophageal eosinophilia and eosinophilic esophagitis (EoE). Am J Gastroenterol 2013; 108: 679-692; quiz 693

[14] Molina-Infante J, Ferrando-Lamana L, Ripoll C et al. Esophageal eosinophilic infiltration responds to proton pump inhibition in most adults. Clin Gastroenterol Hepatol 2011; 9: 110-117

[15] Dellon ES, Speck O, Woodward K et al. Clinical and endoscopic characteristics do not reliably differentiate PPI-responsive esophageal eosinophilia and eosinophilic esophagitis in patients undergoing upper endoscopy: a prospective cohort study. Am J Gastroenterol 2013; 108: $1854-1860$

[16] Moawad FJ, Schoepfer AM, Safroneeva E et al. Eosinophilic oesophagitis and proton pump inhibitor-responsive oesophageal eosinophilia have similar clinical, endoscopic and histological findings. Aliment Pharmacol Ther 2014; 39: 603-608

[17] Warners MJ, van Rhijn BD, Curvers WL et al. PPI-responsive esophageal eosinophilia cannot be distinguished from eosinophilic esophagitis by endoscopic signs. Eur J Gastroenterol Hepatol 2015; 27: 506 511

[18] Iwakura N, Fujiwara Y, Tanaka F et al. Basophil infiltration in eosinophilic oesophagitis and proton pump inhibitor-responsive oesophageal eosinophilia. Aliment Pharmacol Ther 2015; 41: 776 - 784

[19] Spechler SJ, Genta RM, Souza RF. Thoughts on the complex relationship between gastroesophageal reflux disease and eosinophilic esophagitis. Am J Gastroenterol 2007; 102: 1301 - 1306

[20] Dellon ES, Liacouras CA, Molina-Infante J et al. Updated international consensus diagnostic criteria for eosinophilic esophagitis: Proceedings of the AGREE conference. Gastroenterology 2018; 155: 1022 1033

[21] Furuta GT, Liacouras CA, Collins MH et al. Eosinophilic esophagitis in children and adults: a systematic review and consensus recommendations for diagnosis and treatment. Gastroenterology 2007; 133: $1342-1363$

[22] Rodríguez-Sánchez J, Barrio-Andrés J, Nantes Castillejo O et al. The Endoscopic Reference Score shows modest accuracy to predict either clinical or histological activity in adult patients with eosinophilic oesophagitis. Aliment Pharmacol Ther 2017; 45: 300-309

[23] van Rhijn BD, Verheij J, Smout AJ et al. The Endoscopic Reference Score shows modest accuracy to predict histologic remission in adult patients with eosinophilic esophagitis. Neurogastroenterol Motil 2016; $28: 1714-1722$

[24] Dellon ES, Cotton CC, Gebhart JH et al. Accuracy of the eosinophilic esophagitis endoscopic reference score in diagnosis and determining response to treatment. Clin Gastroenterol Hepatol 2016; 14: 31 - 39

[25] Kim HP, Vance RB, Shaheen NJ et al. The prevalence and diagnostic utility of endoscopic features of eosinophilic esophagitis: a meta-analysis. Clin Gastroenterol Hepatol 2012; 10: 988 - 996.e985

[26] Ronkainen J, Talley NJ, Aro P et al. Prevalence of oesophageal eosinophils and eosinophilic oesophagitis in adults: the population-based Kalixanda study. Gut 2007; 56: 615-620

[27] Ma X, Xu Q, Zheng Y et al. Prevalence of esophageal eosinophilia and eosinophilic esophagitis in adults: a population-based endoscopic study in Shanghai, China. Dig Dis Sci 2015; 60: 1716 - 1723

[28] Schoepfer AM, Safroneeva E, Bussmann C et al. Delay in diagnosis of eosinophilic esophagitis increases risk for stricture formation in a time-dependent manner. Gastroenterology 2013; 145: 1230-1236. e1231-1232

[29] Fujiwara Y, Sugawa T, Tanaka F et al. A multicenter study on the prevalence of eosinophilic esophagitis and PPI-responsive esophageal eosinophilic infiltration. Intern Med 2012; 51: 3235 - 3239

[30] Kedika RR, Souza RF, Spechler S]. Potential anti-inflammatory effects of proton pump inhibitors: a review and discussion of the clinical implications. Dig Dis Sci 2009; 54: 2312-2317

[31] Odiase E, Schwartz A, Souza RF et al. New eosinophilic esophagitis concepts call for change in proton pump inhibitor management before diagnostic endoscopy. Gastroenterology 2018; 154: 1217 1221.e1213

[32] Cheng E, Zhang X, Huo X et al. Omeprazole blocks eotaxin-3 expression by oesophageal squamous cells from patients with eosinophilic oesophagitis and GORD. Gut 2013; 62: 824-832

[33] Huo X, Zhang X, Yu C et al. In oesophageal squamous cells exposed to acidic bile salt medium, omeprazole inhibits IL-8 expression through effects on nuclear factor-KB and activator protein-1. Gut 2014; 63: $1042-1052$

[34] Davis BP, Rothenberg ME. Eosinophils and cancer. Cancer Immunol Res 2014; 2: 1-8

[35] Ishibashi S, Ohashi Y, Suzuki T et al. Tumor-associated tissue eosinophilia in human esophageal squamous cell carcinoma. Anticancer Res 2006; $26: 1419-1424$ 